\title{
A new approach to non-commutative inflation
}

\author{
Massimiliano Rinaldi \\ Départment de Physique Théorique, Université de Genève, \\ 24 quai E. Ansermet CH-1211 Genève 4, Switzerland. \\ E-mail: massimiliano.rinaldi@unige.ch
}

\begin{abstract}
We propose an inflationary scenario inspired by a recent formulation, in terms of coherent states, of non-commutative quantum field theory. We consider the semiclassical Einstein equations, and we exploit the ultraviolet finiteness of the non-commutative propagator to construct the expectation value of the energy momentum tensor associated to a generic scalar field. It turns out that the latter is always finite and dominated by an effective cosmological constant. By combining this general feature with the intrinsic fuzziness of spacetime, we show that noncommutativity governs the energy density of the early Universe in such a way that the strong energy condition is violated. Thus, there might be a bounce and a subsequent inflationary phase, which does not need any ad hoc scalar field.
\end{abstract}

PACS numbers: 98.80.Cq, 04.60.Bc

\section{Introduction}

The standard theory of big bang has achieved many successes, from explaining the observed thermal cosmic microwave background (CMB), to the expansion of the Universe and the abundance of light elements. However, it is an incomplete theory as it does not explain why the Universe is almost flat and so homogeneous on large scales. The inflationary paradigm was originally introduced to answer successfully these questions [1. In addition, inflationary theories predicted the tiny fluctuations in the CMB, confirmed later on by observations with increasing accuracy [2.

By definition, inflation consists in a period of accelerated expansion of the Universe, during which gravity acts as a repulsive force. In the simplest inflationary model, this effect is achieved if the dynamics is dominated by a minimally coupled scalar field, called inflaton. With this hypothesis, the strong energy condition is violated, and the energy density behaves as a weakly time-dependent and positive cosmological constant. At the end of inflation, the inflaton decays into radiation and matter according to various reheating mechanisms 3 .

In this paper we investigate the possibility that the inflationary phase is not driven by a classical scalar field, but it rather originates from a non-commutative structure of spacetime, which governs the dynamics through quantum effects encoded in the expectation value of the stress tensor. The idea that non-commutativity can drive the dynamics of the early Universe through its influence on the matter fields is not new, and it was utilized in many important works, see e.g. 4].

According to the semiclassical picture, the Einstein equations are given by

$$
R_{\mu \nu}-\frac{1}{2} R g_{\mu \nu}=8 \pi G\left\langle T_{\mu \nu}\right\rangle,
$$


where $\left\langle T_{\mu \nu}\right\rangle$ is the expectation value of the energy momentum tensor associated to the matter fields. In general, this quantity is divergent but it can be formally written by acting with a differential operator on the propagator, expressed as a function of two distinct points [5]. For example, for a minimally coupled scalar field of mass $m$, and in Euclidean spacetime, we can write

$$
\begin{aligned}
\left\langle T_{\mu \nu}\left(x, x^{\prime}\right)\right\rangle & =\frac{1}{2}\left(g_{\mu}^{\alpha^{\prime}} \nabla_{\alpha^{\prime}} \nabla_{\nu}+g_{\nu}^{\alpha^{\prime}} \nabla_{\mu} \nabla_{\alpha^{\prime}}\right) G_{E}\left(x, x^{\prime}\right)+ \\
& -\frac{1}{2} g_{\mu \nu}\left(g^{\alpha^{\prime} \beta} \nabla_{\alpha^{\prime}} \nabla_{\beta}+m^{2}\right) G_{E}\left(x, x^{\prime}\right),
\end{aligned}
$$

where primed indices refer to $x^{\prime}$, and $G_{E}\left(x, x^{\prime}\right)$ is the Euclidean propagator [6]. In the coincident limit $x=x^{\prime}$ the propagator diverges, and so it does $\left\langle T_{\mu \nu}\right\rangle$. Generally speaking, the singularity structure of the two-point function can always be cast in the Hadamard form $[7]$

$$
G\left(x, x^{\prime}\right)=\frac{u\left(x, x^{\prime}\right)}{\sigma\left(x, x^{\prime}\right)}+v\left(x, x^{\prime}\right) \ln \left|\sigma\left(x, x^{\prime}\right)\right|+w\left(x, x^{\prime}\right),
$$

where $\sigma\left(x, x^{\prime}\right)$ is half the square geodesic distance between $x$ and $x^{\prime}$, and $u, v$, and $w$ are finite functions in the coincident limit [5]. The usual renormalization procedure brings along the addition of higher-curvature terms in the gravitational action, and the Einstein equations become extremely complicated, see e.g. 8 for the cosmological case. Moreover, the right-hand-side of Eq. (1) might contain also a stochastic contribution when the fluctuations of the stress tensor are large [9], but we will not address this issue here.

This situation might change if we modify sensibly the Hadamard form of the propagator. For example, one can introduce a minimal length $L_{P}$ via path-integral duality as in [10. Then, the Euclidean Feynman propagator acquires a regulating term in the Schwinger representation, and takes the form $G\left(x, x^{\prime}\right)=\left(\sigma\left(x, x^{\prime}\right)+L_{P}^{2}\right)^{-1}$. Connections between this proposal and string T-duality were investigated in [11.

Another way of introducing a minimal length is via non-commutativity of spacetime [12]. Implementations of non-commutativity in inflationary models were initially proposed by Brandenberger et al. [13, and Lizzi et al. [14. In these works, the effects of the non-commutativity of spacetime is encoded in the Moyal product, which replaces the ordinary product between functions in the Lagrangian of the inflaton field.

In this paper, we consider an alternative approach to non-commutativity, based on the coordinate coherent states proposed in [15]. In particular, we would like to study what happens to the inflationary phase, when the intrinsic fuzziness of spacetime is expressed not by the Moyal product but by the Gaussian smearing of the source term in the Einstein equations, which is a direct effect of coherent state non-commutativity. In the next section, we will briefly review the main properties of the coordinate coherent states, and we will calculate the stress tensor of a scalar field via semiclassical arguments. In Sec. (3), we will show how the energy density is modified by non-commutative effects, and how this leads to a non-singular solution to the Friedmann equations. In Sec. (4) we study the case when also momenta are noncommuting, and how this can alter the value of the cosmological constant significantly. In the final section, we will discuss further aspects of this model, and the necessary steps to take in order to verify the consistency with current observations. 


\section{Coherent states and non-commutativity}

The proposal put forward in [15] introduces two novelties, with respect to the usual non-commutative scenario. First, all coordinate operators (and not only a subset of) must satisfy the relation $\left[\hat{z}^{\mu}, \hat{z}^{\nu}\right]=i \Theta^{\mu \nu}$, where $\Theta^{\mu \nu}$ is a constant and antisymmetric tensor. In four Euclidean dimensions, one can transform this tensor in a block diagonal form, such that $\Theta^{\mu \nu}=\operatorname{diag}\left(\theta_{1} \varepsilon^{i j}, \theta_{2} \varepsilon^{i j}\right)$, where $\varepsilon^{i j}$ is the two-dimensional Levi-Civita tensor. It turns out that, if $\theta_{1}=\theta_{2}$, the resulting field theory is covariant. The second novelty is that physical coordinates are commuting c-numbers, constructed as expectation values on coherent states. For example, on the Euclidean plane we have two coordinate operators, which satisfy the algebra $\left[\hat{z}_{1}, \hat{z}_{2}\right]=i \theta$. In complete analogy with the case of the harmonic oscillator, where the roles of the position and the momentum operator are now played by the two coordinate operators, one can construct the ladder operators

$$
\hat{A}=\hat{z}_{1}+i \hat{z}_{2}, \quad \hat{A}^{\dagger}=\hat{z}_{1}-i \hat{z}_{2},
$$

such that $\left[\hat{A}, \hat{A}^{\dagger}\right]=2 \theta$. The coherent states $|\alpha\rangle$ are defined as the ones which satisfy the equation $\hat{A}|\alpha\rangle=\alpha|\alpha\rangle$. Finally, the physical coordinates are the commuting cnumbers

$$
x_{1}=\operatorname{Re}(\alpha)=\left\langle\alpha\left|\hat{z}_{1}\right| \alpha\right\rangle, \quad x_{2}=\operatorname{Im}(\alpha)=\left\langle\alpha\left|\hat{z}_{2}\right| \alpha\right\rangle .
$$

Thus, on the non-commutative plane, the vector $\left(x_{1}, x_{2}\right)$ represents the mean position of a point-particle. We note that coherent states are not orthogonal, and form an over-complete basis [16]. In fact, if $|\alpha\rangle$ and $|\beta\rangle$ are coherent states, we have that

$$
\langle\alpha \mid \beta\rangle=\exp \left(\alpha^{*} \beta-\frac{1}{2}|\alpha|^{2}-\frac{1}{2}|\beta|^{2}\right) .
$$

From this, it follows that any operator $\hat{T}$ has the expectation value

$$
\langle\alpha|\hat{T}| \beta\rangle=\exp \left(-\frac{1}{2}|\alpha|^{2}-\frac{1}{2}|\beta|^{2}\right) T\left(\alpha^{*}, \beta\right),
$$

where the function $T\left(\alpha^{*}, \beta\right)$ is defined as the double sum

$$
T\left(\alpha^{*}, \beta\right)=\sum_{n, m} \frac{T_{n m}}{\sqrt{n ! m !}}\left(\alpha^{*}\right)^{n} \beta^{m},
$$

which is well defined and convergent, see [16] for the proof. In the case of the wave operator $\exp \left(i p_{1} \hat{z}_{1}+i p_{2} \hat{z}_{2}\right)$, where $p_{1}$ and $p_{2}$ are the ordinary and commuting components of the momentum, one can easily compute the expectation value by using the Baker-Campbell-Hausdorff formula, and the result is [15]

$$
\left\langle\alpha\left|e^{i p_{1} \hat{z}_{1}+i p_{2} \hat{z}_{2}}\right| \alpha\right\rangle=\exp \left[i \vec{p} \cdot \vec{x}-\frac{\theta}{4}\left(p_{1}^{2}+p_{2}^{2}\right)\right] .
$$

The Gaussian damping is the key feature of this approach to non-commutativity. The same damping enters in the integral measure of the Fourier transform operator, and from this one can construct the quantized theory of a scalar field, see e.g. 17. The above construction can be lifted to higher and even-dimensional spacetimes, and the propagator in a four-dimensional Euclidean spacetime for a scalar field reads

$$
G_{E}(p)=\frac{e^{-p^{2} \theta / 4}}{p^{2}+m^{2}}
$$


In coordinate space, the above propagator is manifestly finite in the ultraviolet regime. In the massless limit, it takes the form [18]

$$
G_{E}\left(x, x^{\prime}\right)=\frac{1-e^{-\sigma_{E}\left(x, x^{\prime}\right) / 4 \theta}}{4 \pi^{2} \sigma_{E}\left(x, x^{\prime}\right)},
$$

where $\sigma_{E}$ is the Euclidean geodesic distance. In the light of Eq. (2), we therefore expect that also $\left\langle T_{\mu \nu}\right\rangle$ will be modified by the non-commutative structure of spacetime, and we aim to investigate what this implies in the inflationary context.

For simplicity, we consider a minimally coupled massive scalar field, which can be interpreted as an effective matter field, or as the field generated by vacuum fluctuations, with the mass acting as an infrared regulator. First, we need to find an expression for the Euclidean propagator in curved space, and the simplest way is to use the proper-time Schwinger representation

$$
G_{E}\left(x, x^{\prime}\right)=\int_{0}^{\infty} d s K\left(x, x^{\prime} ; s\right)
$$

where $K=\left\langle x\left|e^{-i \hat{H} s}\right| x^{\prime}\right\rangle$ and $\hat{H}$ is the Klein-Gordon operator [5]. In [19] it is shown that the modified kernel can be written as $\exp \left(\theta \square_{x}\right) K_{0}\left(x, x^{\prime} ; s\right)$, where $K_{0}$ represents the kernel of the usual theory. As a result, at coincident points we have

$$
K(x ; s)=\frac{e^{-m^{2} s}}{16 \pi^{2}(s+\theta)^{2}}\left[1+e^{[s \theta /(s+\theta)] \square} \sum_{n=1}^{\infty} s^{n} a_{n}(x)\right],
$$

where the deWitt-Schwinger coefficients $a_{n}(x)$ are combination of the Riemann tensor and its derivatives up to order $2 n$, see also [20]. Because of the minimal distance $\sqrt{\theta}$, the integral $(12)$ is no longer singular and can be easily evaluated. By expanding the result for small $m$, we find that

$$
16 \pi^{2} G_{E}(x, x)=\frac{a_{0}}{\theta}-F_{1}\left(\theta m^{2}\right) a_{1}(x)+\cdots,
$$

where the dots indicate terms proportional to $a_{2}$ and $\square a_{1}$ and $F_{1}=1+\gamma+\ln \left(\theta m^{2}\right)+$ $\mathcal{O}\left(\theta m^{2}\right)$. As mentioned above, the mass term acts as an infrared regulator. In this limit, the propagator looks like the one discussed in the case of path-integral duality [10. The other important ingredient is that the Euclidean Green's function satisfies, in curved space, the modified equation

$$
\left(\square_{x}+m^{2}\right) G_{E}(x, y)=e^{\theta \square_{x}}\left[\frac{1}{\sqrt{g}} \delta^{(4)}(x, y)\right],
$$

as a result of the modification of the Fourier transform operator, as mentioned above [19. Since the Green's function is finite at coincident points, we can write the expectation value of the stress tensor $(2)$ as

$$
\left\langle T_{\mu \nu}\right\rangle=\nabla_{\nu} \nabla_{\mu} G_{E}(x)-\frac{1}{2} g_{\mu \nu} \lim _{y \rightarrow x} e^{\theta \square_{x}}\left[\frac{\delta^{(4)}(x, y)}{\sqrt{g}}\right] .
$$

One can show that, for small $(y-x)^{2} / \theta$, we have the general formula [19]

$$
e^{\theta \square_{x}}\left[\frac{\delta^{(4)}(x, y)}{\sqrt{g}}\right]=\frac{1}{16 \pi^{2} \theta^{2}} e^{-(x-y)^{2} / 4 \theta}+\cdots
$$

where the dots stand for terms proportional to powers of $R$, the Ricci scalar. From Eq. (14), we see that the term $\nabla_{\mu} \nabla_{\nu} G_{E}$ is of adiabatic order four and higher. Thus, we 
conclude that the leading term of the deWitt-Schwinger expansion of the Euclidean stress tensor for a scalar field has the form

$$
\left\langle T_{\mu \nu}\right\rangle \simeq \frac{1}{32 \pi^{2} \theta^{2}} g_{\mu \nu} .
$$

This result is general and can be extended to higher spin fields. The non-commutative structure of spacetime naturally regulates the divergent ultra-violet behaviour of the propagator, so the stress tensor for matter fields is UV finite, and the leading term assumes the form of an effective cosmological constant, which diverges in the $\theta \rightarrow 0$ limit. That the cosmological constant is proportional to $\theta^{2}$ is not a surprise, as this is often the case when one introduces a UV cut-off $\theta$ as, for example, in effective field theories 21].

\section{Inflationary phase}

The main lesson learnt from these considerations is that when a minimal length is introduced as in Eq. (13), there is a smearing effect on the expectation value of operators acting on the field. As a result, the quantum stress tensor has a universal form to the leading term.

It has been argued that the smearing effect of the non-commutative geometry can be encoded by replacing the mass term $M$ of the Schwarzschild solution, with a Gaussian distribution [22]

$$
M \delta(r) \longrightarrow \rho(r)=\frac{M}{(4 \pi \theta)^{3 / 2}} e^{-r^{2} / 4 \theta},
$$

where $r$ is the radial coordinate. The Gaussian function is then interpreted as the energy density of a conserved perfect fluid, with radial and tangential pressures. As a result, the Schwarzschild solution is modified into a non-singular solution, which matches asymptotically the usual one. Very recently, this formula was somewhat justified in terms of the Voros product, which is a relative of the star-product, and leads to a field theory that is equivalent to the one displayed above [23], see also [24].

In this section, we would like to find an analogous formula in the case of a Robertson-Walker spacetime. As we are not able to calculate exactly the expression on the right hand side of Eq. (16) in curved spacetime, we use a sort of loop expansion. According to Eq. (7), if $\hat{\rho}$ represents the energy density operator acting on coherent states, one finds that

$$
\langle\hat{\rho}\rangle=\left\langle\alpha\left|\hat{\rho}\left(\hat{z}_{1}, \hat{z}_{2}\right)\right| \alpha\right\rangle=e^{-|\alpha|^{2}} \rho\left(\alpha, \alpha^{*}\right),
$$

and, in the case when $\hat{\rho}=\rho_{0}=$ const,

$$
\langle\hat{\rho}\rangle=e^{-\left(x_{1}^{2}+x_{2}^{2}\right) / 4 \theta} \rho_{0} .
$$

In our case, we consider the result $(18)$ as the "tree level" approximation, and the 00-component of this expression gives the constant energy density $\rho_{0}$. To obtain the energy density at one loop, we promote this constant to the tree-level energy density operator and we let it act on coherent states as in Eq. 211. In doing so, we must recall the global symmetries imposed by a homogeneous and isotropic RobertsonWalker background, that allows only for a time-dependent energy density. As a result of these considerations, we argue that the expression of the energy density to first order has the form

$$
\langle\hat{\rho}\rangle=\rho_{0} e^{-\left(t-t_{0}\right)^{2} / 4 \theta},
$$


where $t_{0}$ is the origin of the time axis and $\rho_{0}=\left(32 \pi^{2} \theta^{2}\right)^{-1}$. Had we imposed spherical symmetry instead, we would have found Eq. 19 , provided we identify the black hole mass as the energy density, i.e. the 00-component of Eq. 18p. We recall that we are treating the semiclassical version of the Einstein equations, in the sense that we restrict the quantum effects on the right-hand side of these, as in Eq. (1). The exact treatment of the problem would involve the calculation of the expectation value the Einstein tensor over coherent states.

If we consider the above equation as a good description of the quantum effects on the energy density, the Friedmann equation takes the form (we choose $t_{0}=0$ )

$$
\left(\frac{\dot{a}}{a}\right)^{2}=\frac{8 \pi G}{3} \rho(t) \equiv H_{0}^{2} e^{-t^{2} / 4 \theta},
$$

where $H=\dot{a} / a$ and $a$ is the scale factor of the metric $d s^{2}=-d t^{2}+a^{2}(t) \delta_{i j} d x^{i} d x^{j}$. As the energy density is no longer singular, we can extend $t$ from $+\infty$ to $-\infty$. Then, the solution to Eq. (23) reads

$$
a(t)=a_{0} \exp \left[H_{0} \sqrt{2 \pi \theta} \operatorname{Erf}\left(\frac{t}{2 \sqrt{2 \theta}}\right)\right],
$$

where

$$
\operatorname{Erf}\left(x^{\prime}\right)=\frac{2}{\sqrt{\pi}} \int_{0}^{x^{\prime}} e^{-x^{2}} d x
$$

and $a_{0}$ is an integration constant. It can be checked that $\ddot{a}$ is initially positive and then changes sign at the time when the comoving Hubble length $(a H)^{-1}$ reaches its minimum, while $H$ reaches a maximum value at $t=0$, see Fig. (1). In other words, the global evolution of the scale factor is very similar to the case of the pre-Big Bang scenario of string cosmology, as $\dot{H}(t)=-\frac{t}{4 \theta H_{0}} H(t)$ [28].

The number of e-folds is $N=\ln \left(a_{f} / a_{i}\right)$, where the asymptotic scale factors are $a_{f, i}=a(t= \pm \infty)$. We find that $N=8 \pi \sqrt{\frac{G \rho_{0} \theta}{3}}$ and, if $N \simeq 60$, this implies that $\sqrt{\theta} \simeq 0.014 \ell_{p}$, where $\ell_{p}$ is the Planck length, and where we assumed that at $t=0$ the value of $\rho_{0}$ is given by the time-time component of Eq. (18). Thus, the minimal length determines uniquely the number of e-folds in the specific case that matter is represented by a single scalar field. In more realistic models, there could be a large number $n$ of other fields that would change the result by a factor $\sqrt{n}$. In any case, the number of e-fold is basically determined by the minimal length of the theory.

The 00-component of the conservation equation for the energy-momentum tensor, yields $\dot{\rho}+3 H(\rho+p)=0$, that can be used to find the effective pressure $p$. If we assume an equation of state of the form $p(t)=\omega(t) \rho(t)$, we find that

$$
\omega \simeq-1+\frac{t}{6 \theta H_{0}}+\mathcal{O}\left(\frac{t^{3}}{\theta^{2}}\right) .
$$

This leads to a dynamical crossing of the $\omega_{0}=-1$ value at the point where $H(t)$ reaches its maximum.

\section{Effective cosmological constant}

If we interpret the result (18) as a cosmological constant, we incur in the same problem as for standard field theory, namely that it is way too large when compared to the observed one. In fact, if we take $\theta$ to be of the order of the square of the Planck 


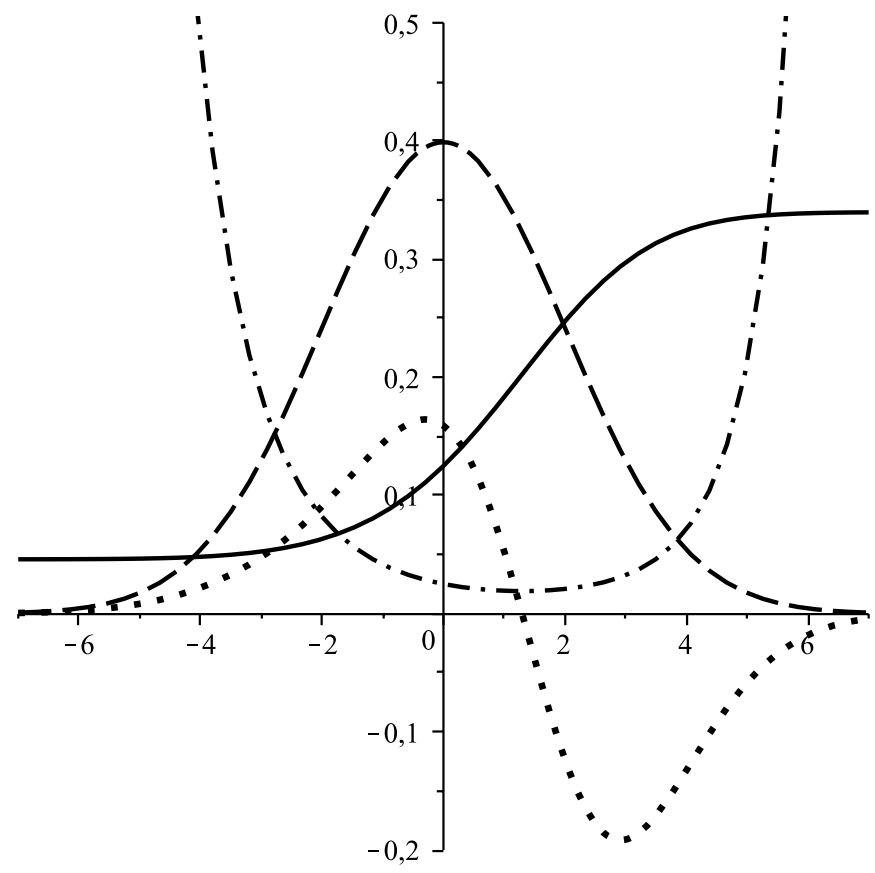

Figure 1. Qualitative behaviour of $a$ (solid line), $\ddot{a}$ (dotted line), $H$ (dashed line), and $(a H)^{-1}$ (dot-dashed line) as functions of time.

length, we would have the usual $10^{120}$ discrepancy problem of the Standard Model [25]. One possibility is that $\theta$ is in fact only a "bare" parameter, that can be rescaled by an effective theory approach, along the lines of [10. Another possibility is that this large quantity can be suppressed by properly defined boundary conditions [26. There is however another intriguing way of dealing with this issue. The parameter $\theta$ can in fact be rescaled if we assume that also momenta do not commute. This hypothesis has been studied in several works (see e.g. [27] and references therein), and it is originally motivated by the interaction with a constant magnetic field $B$ of a particle moving on a non-commutative plane. In such a situation, the algebra of the operators is

$$
\left[\hat{x}_{i}, \hat{x}_{j}\right]=i \theta \varepsilon_{i j}, \quad\left[\hat{p}_{i}, \hat{p}_{j}\right]=i \gamma \varepsilon_{i j}, \quad\left[\hat{x}_{i}, \hat{p}_{j}\right]=i \hbar \delta_{i j},
$$

where $\varepsilon_{i j}$ is the two-dimensional Levi-Civita tensor, and $\gamma$ is a sort of "minimal momentum", which is proportional to $B$. This algebra can be reduced to the one with commuting momenta by rescaling the latter according to

$$
\begin{aligned}
& \hat{p}_{i} \longrightarrow \hat{\pi}_{i}=\alpha \hat{p}_{i}+\beta \varepsilon_{i j} \hat{x}_{j}, \quad \alpha=\frac{1}{\sqrt{1-\theta \gamma}}, \\
& \beta=\frac{1}{\theta}(1-\alpha), \quad\left[\hat{\pi}_{i}, \hat{\pi}_{j}\right]=0 .
\end{aligned}
$$

We remark that, at the critical value $\gamma \theta=1$, the linear transformation above becomes singular. This situation corresponds to a change of the symmetry group acting on the plane, from $\mathrm{SU}(2)$ to $\mathrm{SU}(1,1)$, as explained in [27. In our context, the important fact is that when we compute the expectation value on coherent states of the rescaled wave operator $\exp (i \hat{\overrightarrow{\vec{\pi}}} \cdot \hat{\vec{x}})$, we find that the damping factor is now multiplied by $\alpha$. It 
follows that the energy momentum tensor is modified according to

$$
\left\langle T_{\mu \nu}\right\rangle \sim \frac{1}{\theta^{2} \alpha^{4}} g_{\mu \nu}=\frac{(1-\gamma \theta)^{2}}{\theta^{2}} g_{\mu \nu} .
$$

This shows that the effective cosmological constant can actually be much smaller than previously estimated, if the product $\gamma \theta$ is close to one.

\section{Discussion}

The inflationary phase ends when $\ddot{a}=0$, namely at the time $t_{e}$, implicitly determined by $t_{e}=4 \theta H_{0} e^{-t_{e}^{2} / 8 \theta}$. The subsequent evolution of the Universe should be driven by a matter content other than the right hand side of Eq. 23). In most inflationary models, matter and radiation are generated by the decay of the inflaton. Alternatively, it is known that the expansion of the Universe itself can be a source of particle production 29. As the expansion rate is initially of the order of $H_{0} \sim \theta^{-1 / 2}$, we can expect that the energy density of these particles will be roughly of the order of the GibbonsHawking temperature $T \simeq H_{0} / 2 \pi \sim \theta^{-1 / 2}$ [30. However, particle production could be tamed by the minimal length. In fact, in a recent study on the Unruh effect, based on the propagator (11), it is shown that the thermal spectrum seen by an accelerated detector is strongly suppressed [18. This could be a hint towards excluding particle production as an efficient mechanism to produce matter in an expanding Universe, and further investigations are on their way.

There is another logical option that might render reheating not necessary. In fact, it seems plausible to assume that the Universe can collapse before $t=0$, because it is dominated by some form of (classical) matter. When the energy density grows and reaches planckian values, non-commutative effects take over and drive the Friedmann equations as explained above. In this sense, we can talk about a bouncing solution, in analogy with the pre-Big Bang scenario. In this scenario, the matter that dominates after the non-commutative phase can be a remnant of the primordial matter responsible for the crunch.

Cosmological perturbations in the presence of a bounce have been studied since the early paper 31. From these studies it turns out that quantum vacuum fluctuations, which exit the Hubble horizon during a matter-dominated contracting phase, evolve to form a scale-invariant spectrum today 32. Eventually, a bouncing phase can leave specific non-gaussian signatures in the spectrum [33. In our model, the final contracting phase is dominated by the non-commutative effects. On one hand, one can argue that around the bounce at $t=0$, the Universe expand quasiexponentially and therefore the background equations are indistinguishable from the ones already studied in the literature. It seems therefore plausible to claim that the spectrum of cosmological perturbation is scale invariant and that it might even show non-gaussianity. On the other hand, also perturbations feel the non-commutative structure of spacetime. Therefore, one should analyze the evolution of perturbations, taking in account that the propagator associated to graviton modes must satisfy an equation similar to 15). Again, this could leave distinctive fingerprints in the CMB, and this exciting possibility will be the subject of a future work.

In this paper we described how the non-commutative structure of spacetime can act on the Einstein equations via semiclassical effects, and drive the Universe through an inflationary phase. The semiclassical picture shows that the effect induced by non-commutativity on matter fields smears out the energy density, causing the 
violation of the strong energy condition, and, eventually, a smooth transition between a contracting and an expanding Universe. This phenomenon seems quite general, and it was already noticed in the context of non-commutative black holes.

The inflationary scenario proposed in this work is based on two assumptions. The first is the algebra of the coordinate operators, that reminds the one for the coherent states of quantum mechanics. We mentioned that this model can be justified in terms of the Voros product, which belongs to the same family as the star-product 23. This means that both the star-product and the Voros product are equally valid candidates as the non-commutative algebra. Therefore, assuming the Voros product is no more ad hoc than assuming the star-product as the algebra governing non-commutativity among coordinates.

The second, and perhaps the strongest, assumption made in this paper is that non-commutativity does not affect the left hand side of the Einstein equations as in the semiclassical approximation, where quantum effects are relegated to the source term only. This is common to many other non-commutative models and reflects our ignorance about a consistent way of quantizing the Einstein equations. In our case, we assume that the quantum effects on the source arise from the fuzziness induced by quantum fluctuations at the scale $\theta$. A similar hypothesis is adopted in [4 but we believe that our work has the advantage of going a little further, as it provides a mechanism for a regular bounce.

For these reasons, we believe that our model deserves further investigations. To begin with, it is crucial to determine the spectrum of the primordial fluctuations, an verify its flatness. Also, it is necessary to come up with a precise mechanism of reheating, or determine how matter eventually existing before the non-commutative phase evolves through it. Finally, we should study more deeply the intriguing possibility that the effective cosmological constant arises from a symmetry-breaking at a more fundamental level of the theory, as mentioned in Sec. 4. We hope to report shortly and more accurately on these issues.

\section{Acknowledgments}

I wish to thank M. Arzano, C. Bœehm, R. Durrer, P. Nicolini, and E. Spallucci for many valuable discussions. This work is supported by the Fond National Suisse.

\section{References}

[1] V. F. Mukhanov and G. V. Chibisov, JETP Lett. 33 (1981) 532 [Pisma Zh. Eksp. Teor. Fiz. 33 (1981) 549]; A. A. Starobinsky, Phys. Lett. B 117 (1982) 175.

[2] E. Komatsu et al. [WMAP Collaboration], Astrophys. J. Suppl. 180 (2009) 330.

[3] V. Mukhanov, Physical Foundations of Cosmology, (Cambridge University Press, Cambridge, England, 2005); R. Durrer, The Cosmic Microwave Background (Cambridge University Press, Cambridge, England, 2008).

[4] S. Alexander, R. Brandenberger and J. Magueijo, Phys. Rev. D 67 (2003) 081301.

[5] N. D. Birrell and P. C. W. Davies, Quantum Fields in Curved Space, (Cambridge University Press, London, 1982).

[6] S. M. Christensen, Phys. Rev. D 14 (1976) 2490.

[7] R. M. Wald, Commun. Math. Phys. 54 (1977) 1; S. A. Fulling, M. Sweeny and R. M. Wald, Commun. Math. Phys. 63 (1978) 257.

[8] T. S. Bunch and P. C. W. Davies, J. Phys. A 11 (1978) 1315.

[9] C. I. Kuo and L. H. Ford, Phys. Rev. D 47 (1993) 4510; E. Calzetta and B. L. Hu, Phys. Rev. D 49 (1994) 6636.

[10] T. Padmanabhan, Phys. Rev. D 57 (1998) 6206. 
[11] M. Fontanini, E. Spallucci and T. Padmanabhan, Phys. Lett. B 633 (2006) 627.

[12] H. S. Snyders, Phys. Rev. 71, 38 (1947); A. Connes, Géometrie non commutative, (InterEditions, Paris, France, 1990).

[13] R. Brandenberger and P. M. Ho, Phys. Rev. D 66 (2002) 023517.

[14] F. Lizzi, G. Mangano, G. Miele and M. Peloso, JHEP 0206 (2002) 049.

[15] A. Smailagic and E. Spallucci, J. Phys. A 37 (2004) 1 [Erratum-ibid. A 37 (2004) 7169]; J. Phys. A 36 (2003) L467; A. Smailagic and E. Spallucci, J. Phys. A 36 (2003) L517.

[16] R. J. Glauber, Phys. Rev. 131 (1963) 2766.

[17] M. Rinaldi, Mod. Phys. Lett. A 25 (2010) 2805; R. Casadio, A. Gruppuso, B. Harms and O. Micu, Phys. Rev. D 76 (2007) 025016.

[18] P. Nicolini and M. Rinaldi, Phys. Lett. B 695 (2011) 303.

[19] E. Spallucci, A. Smailagic and P. Nicolini, Phys. Rev. D 73 (2006) 084004.

[20] L. Modesto and P. Nicolini, Phys. Rev. D 81 (2010) 104040.

[21] A. G. Cohen, D. B. Kaplan and A. E. Nelson, Phys. Rev. Lett. 82 (1999) 4971; J. F. Donoghue, Phys. Rev. D 50 (1994) 3874.

[22] P. Nicolini, J. Phys. A 38, L631 (2005); P. Nicolini, A. Smailagic and E. Spallucci, Phys. Lett. B 632 (2006) 547; P. Nicolini, Int. J. Mod. Phys. A 24 (2009) 1229; M. Bleicher and P. Nicolini, J. Phys. Conf. Ser. 237 (2010) 012008.

[23] R. Banerjee, S. Gangopadhyay and S. K. Modak, Phys. Lett. B 686 (2010) 181.

[24] F. G. Scholtz, L. Gouba, A. Hafver and C. M. Rohwer, J. Phys. A 42 (2009) 175303.

[25] S. Weinberg, Rev. Mod. Phys. 61 (1989) 1.

[26] M. Maggiore, arXiv:1004.1782 [astro-ph.CO].

[27] V. P. Nair and A. P. Polychronakos, Phys. Lett. B 505 (2001) 267; S. Bellucci, A. Nersessian and C. Sochichiu, Phys. Lett. B 522 (2001) 345.

[28] M. Gasperini and G. Veneziano, Phys. Rept. 373 (2003) 1.

[29] L. Parker, Phys. Rev. 183 (1969) 1057; Y. B. Zeldovich, Pisma Zh. Eksp. Teor. Fiz. 12 (1970) 443; L. H. Ford, Phys. Rev. D 35 (1987) 2955.

[30] G. W. Gibbons and S. W. Hawking, Phys. Rev. D 15 (1977) 2738.

[31] A. A. Starobinsky, JETP Lett. 30 (1979) 682 [Pisma Zh. Eksp. Teor. Fiz. 30 (1979) 719].

[32] F. Finelli and R. Brandenberger, Phys. Rev. D 65 (2002) 103522; D. Wands, Phys. Rev. D 60 (1999) 023507; L. E. Allen and D. Wands, Phys. Rev. D 70 (2004) 063515; P. Peter and N. Pinto-Neto, Phys. Rev. D 78 (2008) 063506.

[33] Y. F. Cai, W. Xue, R. Brandenberger and X. Zhang, JCAP 0905 (2009) 011. 